Int. J. Odontostomat.,

5(2):203-207, 2011.

\title{
Prevalencia de Historia de Caries en Escolares de 10 Años, Frutillar, 2007-2010
}

\author{
Caries Prevalence in 10 - Year-Old School Children, Frutillar, 2007-2010
}

Andrea Ceron*; Viviana Castillo* \& Pedro Aravena*,**

CERON, A.; CASTILLO, V. \& ARAVENA, P. Prevalencia de historia de caries en escolares de 10 años, Frutillar, 2007-2010. Int. J. Odontostomat., 5(2):203-207, 2011.

RESUMEN: En Chile, la prevalencia de historia de caries dental en niños de 6 y 12 años es de 70,3\% y 62,5\%, respectivamente. Por ello, el Programa de Salud Bucal de la Junta Nacional de Auxilio Escolar y Becas (JUNAEB) desarrolla promoción, prevención y tratamiento dental con el fin de disminuir esta tasa en poblaciones más vulnerables, como en la ciudad de Frutillar. El objetivo de esta investigación fue determinar la prevalencia caries por medio de los índices ceod y COPD en niños de 10 años atendidos en el Modulo Dental JUNAEB de la ciudad de Frutillar entre el año 2007 a 2010. Estudio descriptivo. Se recolectaron los formularios de pacientes de 10 años atendidos en el Modulo dental JUNAEB entre el 1 de enero de 2007 al 31 de diciembre de 2010. Por cada año, se registró el número de pacientes, el género y los índices COPD y ceod. Se evaluó la asociación de los datos demográficos con la historia de caries usando la prueba chi-cuadrado, $t$ de student y ANOVA ( $p<0.05)$. Se registraron 389 pacientes. El 83,03\% presentó historia de caries. El índice promedio COPD y ceod fue de 1,56 (D.E. $\pm 1,8)$ y 1,65 (D.E. $\pm 1,94)$ respectivamente. Se observó una reducción significativa en la historia de caries $(p=0,04)$ y en el índice COPD $(p=0,001)$ en el último año. No se observó una asociación de historia caries con el género ni en el número de pacientes $(p=0,57)$. La prevalencia de historia caries en niños de 10 años disminuyó significativamente entre el año 2007 al 2010. Se promueve realizar estudios de cohorte con el fin de evaluar el efecto real del programa en niños de entre 6 y 12 años de edad.

PALABRAS CLAVE: caries dental, COPD, prevalencia, niños, Chile.

\section{INTRODUCCIÓN}

La caries dental constituye la enfermedad más importante de la práctica odontológica, persistiendo como un importante desafío para la salud pública dada su alta prevalencia, especialmente en países no desarrollados y de alto impacto en la población infantil. La Organización Mundial de la Salud ha estimado que entre el 60 y $90 \%$ de los niños del mundo presentan lesiones de caries con cavitación evidente (Cereceda et al., 2010).

La historia de caries dental es medida a través de los índices COPD (Klein et al., 1938) para dentición permanente y ceod (Gruebbel, 1944) para dentición temporal, siendo recomendados por la OMS (OMS, 1997). Estos índices originalmente fueron creados para describir el estado dental y la necesidad de tratamiento en niños de escuelas primarias (Larmas, 2010).
En Chile, los últimos estudios de prevalencia realizados por el Ministerio de Salud, mostraron la presencia de altos índices de caries en la población infantil, con una prevalencia, en niños(as) de 6 y 12 años, de $70,3 \%$ y $62,5 \%$, respectivamente (Cereceda et al.).

Dada la alta prevalencia de esta patología, ha sido necesario orientar las políticas de salud bucal a grupos de alto riesgo y población vulnerable desarrollando estrategias promocionales y preventivas. Es así, como se ha priorizado la población menor de 20 años para la atención en salud bucal, los que se incluyeron dentro de los objetivos sanitarios para la década 2000 - 2010 (MINSAL, 2000).

Dentro de las medidas adoptadas por el Ministerio de Salud se encuentra el Programa de Salud Bucal

\footnotetext{
Instituto de Odontoestomatología. Universidad Austral de Chile, Valdivia, Chile.

"* Programa Doctorado Ciencias Médicas. Facultad de Medicina. Universidad de La Frontera, Temuco, Chile.
} 
de la Junta Nacional de Auxilio Escolar y Becas (JUNAEB) que consiste en desarrollar acciones de promoción, prevención y recuperación de la salud bucal a estudiantes desde pre-kinder a octavo básico (4 a 14 años), de escuelas municipales y particulares subvencionadas de zonas urbanas y rurales. Dichas atenciones son entregadas en instalaciones clínicas comúnmente denominadas como "Módulos dentales".

La relevancia de realizar un perfil epidemiológico en comunas donde se aplique este programa estatal, como es la ciudad de Frutillar en la X Región de Chile, permite estimar el efecto que éste ha tenido a lo largo del tiempo en niños cuyas edades fluctúen entre los 6 y 12 años, entregándonos bases concretas para poder evaluar y reorientar en función de sus resultados estos programas gubernamentales.

El objetivo de este estudio es determinar la prevalencia caries por medio de los índices ceod y COPD en niños de 10 años atendidos en el Modulo Dental JUNAEB de Frutillar desde el año 2007 a 2010 y el impacto que ha tenido el programa sobre estos índices.

\section{MATERIAL Y MÉTODO}

Estudio descriptivo basado en registros clínicos. En el mes de abril del año 2011 se obtuvo una muestra de los registros dentales diarios correspondientes al formulario F4 de pacientes de 10 años que acudieron a su control y tratamiento dental en el módulo JUNAEB entre Enero del año 2007 y 31 Diciembre del año 2010 de la ciudad de Frutillar, ubicada a 980 kilometros al sur de Santiago y con una población total de 15.403 habitantes. Se excluyeron aquellos registros donde los índices ceod y COPD fueran evaluados en dos o más veces durante los años de registro o aquellos incompletos.

El registro de todos los datos contó con el consentimiento y la aprobación de los familiares de los menores atendidos mediante un formulario de aten- ción presente en el módulo de atención JUNAEB de Frutillar. El análisis y la extracción de los datos desde la base de registros anuales fueron aportados con la autorización de la Directora del Departamento de Salud Municipal de Frutillar.

Por cada año, se registró el número de niños atendidos, el sexo y la historia de caries mediante el índice COPD y ceod. Los resultados se describen a través de promedios con sus respectivas desviaciones estándar y las medidas extremas de cada índice observados por año.

Para establecer la asociación entre la historia de caries con el sexo de los pacientes y el año de atención se usó la prueba chi-cuadrado de Pearson, $t$ de student y ANOVA con un nivel de significancia estadísitica $p<0,05$.

La tabulación de los datos se realizó con el software MS Excel 2003 (Microsoft, USA) y el análisis con el paquete estadístico Stata 10.0 (StataCorp LP. USA).

\section{RESULTADOS}

En los registros seleccionados se atendieron a 389 pacientes, siendo 201 mujeres $(51,67 \%)$. De todos ellos, 323 (83,03\%) presentaron historia de caries. En el año 2007 se observó una prevalencia de historia de caries de 83,1\% y en el año 2010 un 72,37\%, observándose una reducción estadísticamente significativa en la historia de caries en el último año $(p=0,04)$ (Fig. 1). No se observó una asociación de historia caries con el género ni en el total de pacientes observados por año $(p=0,57)$ (Tabla I).

El valor promedio del índice COPD fue de 1,56 (D.E. $\pm 1,8)$ e índice ceod fue de 1,65 (D.S. $\pm 1,94)$ (Tablas II y III). Se observó una disminución estadísticamente significativa en la evolución del promedio COPD entre los años 2007 y el $2010(p=0,001)$.

Tabla I. Prevalencia de historia de caries en niñas y niños de 10 años según el sexo. Modulo Dental JUNAEB de Frutillar.

\begin{tabular}{lcccc}
\hline Año & Hombre $(\boldsymbol{\%})$ & Mujer $(\boldsymbol{\%})$ & Total & valor $\mathbf{p}$ \\
\hline $\mathbf{2 0 0 7}$ & $28(47,46)$ & $31(52,54)$ & 59 & 0.87 \\
$\mathbf{2 0 0 8}$ & $50(44,25)$ & $63(55,75)$ & 113 & 0.11 \\
$\mathbf{2 0 0 9}$ & $51(53,12)$ & $45(46,88)$ & 96 & 0.48 \\
$\mathbf{2 0 1 0}$ & $25(45,45)$ & $30(54,55)$ & 55 & 0.86 \\
General & $154(47,68)$ & $169(52,32)$ & 323 & 0,57 \\
\hline
\end{tabular}




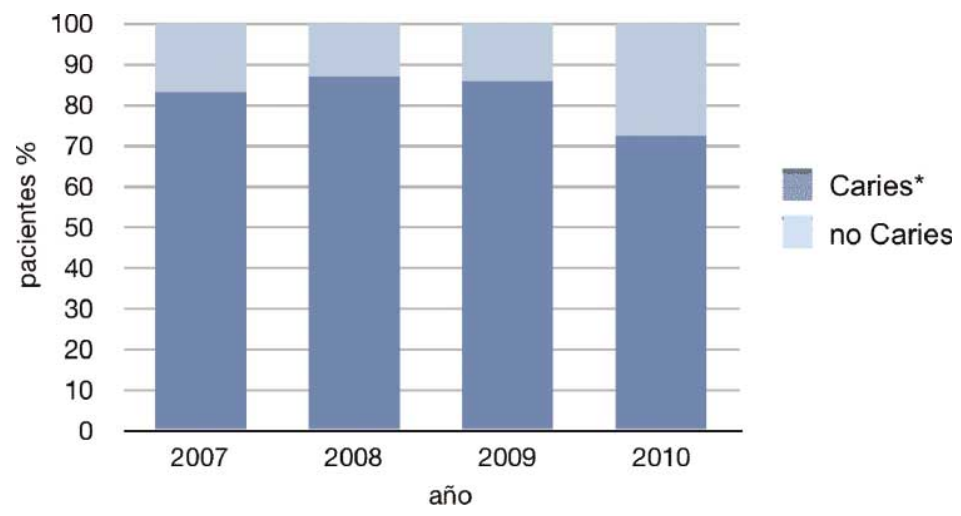

Fig. 1. Porcentaje de pacientes con historia de caries según año. Modulo Dental JUNAEB de Frutillar. *p:0.04 (chi-cuadrado de Pearson)

Tabla II. Promedio de índice COPD en niñas y niños de 10. Modulo Dental JUNAEB de Frutillar.

\begin{tabular}{lccc}
\hline Año & COPD (D.E.) & min - $\mathbf{m a x}$ & valor $\mathbf{~ * *}$ \\
\hline $\mathbf{2 0 0 7}$ & $2,09(2,19)$ & $0-8$ & - \\
$\mathbf{2 0 0 8}$ & $1,60(1,79)$ & $0-9$ & 0,36 \\
$\mathbf{2 0 0 9}$ & $1,59(1,64)$ & $0-8$ & 0,38 \\
$\mathbf{2 0 1 0}$ & $0,96(1,45)$ & $0-7$ & 0,001 \\
General & $1,56(1,80)$ & $0-9$ & - \\
${ }^{*}$ En base al año 2007. Prueba ANOVA -Bonferroni.
\end{tabular}

Tabla III. Promedio de índice ceod en niñas y niños de 10. Modulo Dental JUNAEB de Frutillar.

\begin{tabular}{lccc}
\hline Año & ceod (D.E.) & min - $\mathbf{m a x}$ & valor $\mathbf{p}^{*}$ \\
\hline $\mathbf{2 0 0 7}$ & $1,32(1,77)$ & $0-7$ & - \\
$\mathbf{2 0 0 8}$ & $1,90(2,04)$ & $0-8$ & 0,25 \\
$\mathbf{2 0 0 9}$ & $1,46(1,77)$ & $0-8$ & 1 \\
$\mathbf{2 0 1 0}$ & $1,80(2,13)$ & $0-7$ & 0,81 \\
General & $1,65(1,94)$ & $0-8$ & - \\
\hline
\end{tabular}

*En base al año 2007. Prueba ANOVA -Bonferroni.

\section{DISCUSIÓN}

La prevalencia de historia de caries entre los años 2007 y 2010 fue de $83,03 \%$. Considerando que es una población urbano-rural los datos estadísticos se enmarcan dentro de los resultados basales aportados por distintas instancias del Ministerio de Salud: En un primer reporte el año 1996 Urbina et al. (1996) demuestran una tasa de niños libres de caries de $14,1 \%$ entre los 6 a 8 años y un $19,9 \%$ a los 12 años de edad.

Los últimos estudios de prevalencia liderados del Ministerio de Salud en el año 2007 y 2010 mostraron la presen- cia de altos índices de caries en la población infantil, con una prevalencia, en niños (as) de 6 y 12 años, de $70,3 \%$ y 62,5\%, y un índice promedio de ceod y COPD de 3,7 y 1,9 respectivamente (MINSAL, 2007a, b).

La tasa obtenida en esta investigación se enmarca en una población urbano-rural que, por el objetivo del programa JUNAEB, se enfoca principalmente a grupos poblacionales de escasos recursos. Resultados similares al informe otorgado por Cereceda et al. quienes mostraron una prevalencia de historia de caries de $79,5 \%$ en una muestra estratificada de niños entre 5 y 15 años pertecientes a comunidades de ingreso socioeconómico medio-bajo y bajo de la ciudad de Santiago.

En Peralillo, localidad rural en Chile, la prevalencia global de caries dental en niños de 4 a 5 años de edad fue de 49,2\% (Rivera, 2011), valores que pueden contrastarse con los descritos por Vargas \& Herrera (2002) donde en las comunidades indígenas mapuches de extrema ruralidad, nivel socioeconómico bajo y de escasa oferta asistencial el $99 \%$ de niños entre 6 y 12 años presentaron historia de caries.

El índice COPD ha mostrado una disminución desde el año 2007 al 2010, siendo esta diferencia estadísticamente significativa $(p<0,05)$. En otros casos no se ha visto reflejada esta diferencia ante una intervención clínica, pero si la calidad de salud oral expresada en el aumento de la tasa de dientes obturados y una disminución del número de caries expresadosn en las variables $\mathrm{O}$ y $\mathrm{C}$ del índice COPD, respectivamente (Labranque \& Vidal, 2001). Este cambio a través del tiempo es el resultado a la implementación de medidas preventivas en base a flúor y programas gubernamentales de prevención y educación de la población más expuesta a los factores de riesgo de la caries (Mendez \& Caricote, 2003).

El programa de salud bucal de JUNAEB se implementó en el año 2006 en la ciudad de Frutillar, por lo que puede verse reflejado en la disminución estadísticamente significativa de las tasas de historia de caries y en la disminución de los índices COPD en el año 2010. Sin embargo, es importante destacar las limitacio- 
nes de este estudio, como son la edad de registro de los índices de historia de caries y la forma de recolección de datos que podrían ser fuente de un sesgo del observador, debido a que durante el período del año 2007 al 2010 participó una odontóloga a cargo del programa que contó con la colaboración de alumnos(as) internos de la carrera odontología provenientes de la Universidad de Concepción y de la Universidad Austral de Chile. Además, en estas estadísticas, no existe un registro diferenciado de la población urbana o rural, donde la población rural no tiene acceso a agua potable fluorada y no consideran el riesgo social de cada paciente, lo cual puede influir en los índices ceod y COPD (MINSAL, 2007a, b).

En conclusión, la prevalencia de historia caries y los índices de COPD en niños de 10 años atendidos en el Módulo JUNAEB de la ciudad de Frutillar ha disminuido significativamente desde el año 2007 al 2010 sin diferencias de sexo.
Se postula un estudio de tipo corte transversal, diferenciando la población urbana de la rural. También sería recomendable realizar una investigación de cohorte prospectiva que evalúe la situación bucal de los niños atendidos en el módulo dental desde su ingreso (Pre-kinder) hasta la finalización del programa (Octavo Básico), con odontológos calibrados en el transcurso del seguimiento.

\section{AGRADECIMIENTOS}

Agradecemos a la Dra. Sandra Álvarez encargada Módulo Dental JUNAEB de Frutillar; a la Señora Pamela Gopaldas, Directora Departamento Salud Frutillar por las facilidades y el apoyo a este trabajo de investigación y al Dr. Ricardo Cartes del programa de Doctorado de Ciencias Médicas por su aporte en la revisión y comentarios finales del documento.

CERON, A.; CASTILLO, V. \& ARAVENA, P. Caries prevalence in 10-year-old school children, Frutillar, 2007-2010. Int. J. Odontostomat., 5(2):203-207, 2011.

ABSTRACT: In Chile, the prevalence of dental caries history in children aged 6 and 12 years is $70.3 \%$ and $62.5 \%$ respectively. Therefore, the Oral Health Program of the National Board of Student Aid and Scholarships (JUNAEB) carry out promotion, prevention and dental treatment to reduce this rate in more vulnerable populations, such as in Frutillar schoolchildren. The aim was to determine caries prevalence using dmft and DMFT rates in 10-year-old children treated at the JUNAEB Dental Module in Frutillar between 2007 and 2010. Descriptive study. Records were collected from 10 years old patients treated at the JUNAEB Dental Module between January 1, 2007 to December 31, 2010. For each year, we registered the number of patients, sex and DMFT/dmft index. We evaluated the association of demographics with the history of caries using the chi-square test, $t$ test and ANOVA $(p<0.05)$. There were 389 patients. $83.03 \%$ had a history of dental caries. The average DMFT and dmft was $1.56(\mathrm{SD} \pm 1.8)$ and $1.65(\mathrm{SD} \pm 1.94)$ respectively. There was a significant reduction in dental caries history $(p=0.04)$ and DMFT index $(p=0.001)$ in the last year. There was no association between dental caries history and sex or number of patients $(p=0.57)$. The prevalence of of 10 -year-old dental caries history decreased significantly between 2007 and 2010. Ongoing cohort studies are warranted to assess the actual effect of the program in children between 6 and 12 years old. .

KEY WORDS: dental caries, dmft index, prevalence, children, Chile.

\section{REFERENCIAS BIBLIOGRÁFICAS}

Cereceda, M. M. A.; Simone, F. C.; Ormeño, Q. A.; Pinto, G. M.; Tapia, V. R.; Díaz, S. C. \& García, B. H. Prevalencia de caries en alumnos de educación básica y su asociación con el estado nutricional. Rev. Chil. Pediatr., 81(1):28-36, 2010.

Gruebbel, A. O. A measurement of dental caries prevalence and treatment service for deciduous teeth. J. Dent. Res., 23:163-8, 1944.

Klein, H.; Palmer, C. E. \& Knutson, J. W. Studies on
Dental Caries, I, Dental status and Dental Needs of elementary school. Pub. Health Rep., 53:751$65,1938$.

Labranque, C. R. \& Vidal, V. H. Estudio comparativo de salud oral en una población escolar rural de la VI Región. Rev. Dent. Chile, 92(1):13-6, 2001.

Larmas, M. Has dental caries prevalence some connection with caries index values in adults? Caries Res., 44(1):81-4, 2010. 
Méndez, D. \& Caricote, N. Prevalencia de caries dental en escolares de 6 a 12 años de edad del municipio Antolín del Campo, estado Nueva Esparta, Venezuela (2002-2003). Revista Latinoamericana de Ortodoncia y Odontopediatria, 2003. Disponible en: http://www.ortodoncia.ws/publicaciones/ 2003/caries_dental_escolares.asp

MINSAL. Lineamientos estratégicos. Plan nacional de salud bucal 2000-2010. Chile, 2000. Disponible en: http://www.redsalud.gov.cl/portal/url/page/minsalcl/

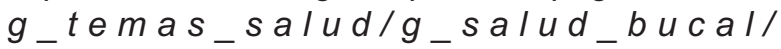
salud_bucal_temas_salud.html

MINSAL; Ceballos, M.; Acevedo, C.; Corsini, G. \& Jans, A. Diagnóstico de Salud Bucal de Niños de 2 y 4 años, que asisten a la educación preescolar en la Región Metropolitana, 2007a. Disponible en: http:/ /www.minsal.gob.cl/portal/url/item/ 7dc33dfObb34ec58e04001011e011c36.pdf

MINSAL; Soto, L.; Tapia, R.; Jara, G. \& Rodríguez, G. Diagnóstico Nacional de Salud Bucal del Niño de 6 años, 2007b. Disponible en: http:// www.minsal.gob.cl/portal/url/item/ 7dc33df0bb34ec58e04001011e011c36.pdf

Organización Mundial de la Salud (OMS). Encuestas de salud bucodental. Métodos básicos. Ginebra, Suiza, Organización Mundial de la Salud, 1997.

Rivera, M. C. A. Pre-school child oral health in a rural Chilean community. Int. J. Odontostomat., 5(1):836, 2011.

Urbina, T.; Caro, J. C. \& Vicent, M. Caries dentaria y fluorosis en niños de 6, 8 y 12 años de la II, VI, VIII, IX, X y RM. Chile 1996. Santiago, División Programas de Salud, Departamento Odontológico MINSAL, 1996.

Vargas, A. R. \& Herrera, C. M. Estudio de prevalencia de caries en escolares de las comunidades rurales mapuches de Panguinilague, Puquiñe y Lago Neltume. Provincia de Valdivia. $X$ región de Los Lagos. Rev. Dent. Chile, 93(3):3-8, 2002.
Dirección de correspondencia:

Pedro Aravena Torres

Instituto de Odontoestomatología

Facultad de Medicina

Universidad Austral de Chile

Rudloff 1640

Valdivia

CHILE

Fono: (56-63) 293751

Email: paravena@uach.cl

Recibido : 05-08-2011

Aceptado: 23-08 2011 